\title{
STUDIES OF CORONAVIRUS DI RNA REPLICATION USING IN VITRO CONSTRUCTED DI CDNA CLONES
}

\author{
Shinji Makino and Michael M.C. Lai \\ Department of Microbiology, University of Southern California, School of Medicine \\ Los Angeles, CA 90033
}

\begin{abstract}
Sequence analysis of an intracellular defective-interfering (DI) RNA, DIssE, of mouse hepatitis virus (MHV) revealed that it is composed of three noncontiguous genomic regions, representing the first 864 nucleotides of the 5'-end, an internal 748 nucleotides of the polymerase gene, and 601 nucleotides from the 3'end of the parental MHV genome. DIssE had three base substitutions within the leader sequence and also a deletion of nine nucleotides located at the junction of the leader and the remaining genomic sequence. A system was developed for generating DI RNAs to study the mechanism of MHV RNA replication. A cDNA copy of DIssE RNA was placed downstream of T7 RNA polymerase promoter to generate DI RNAs capable of extremely efficient replication in the presence of a helper virus. We demonstrated that, in the DI RNAtransfected cells, the leader sequence of these DI RNAs was switched to that of the helper virus during one round of replication. This high-frequency leader sequence exchange was not observed if a nine-nucleotide stretch at the junction between the leader and the remaining DI sequence was deleted. This observation suggests that a free leader RNA is utilized for the replication of MHV RNA.
\end{abstract}

\section{INTRODUCTION}

Mouse hepatitis virus (MHV) contains a single-stranded infectious RNA genome of more than 32 kilobases (kb) (11; Shieh, C.-K. and Lai, M. M. C., unpublished data). It synthesizes in infected cells six major species of subgenomic mRNAs, which have a 3'-coterminal, nested-set structure $(7,12,20)$ and contain an identical 5'-end leader sequence of $72-77$ nucleotides $(6,9,25)$. A considerable body of evidence suggests that MHV utilizes a leader-primed transcription mechanism, in which a leader RNA transcribed from the $3^{\prime}$-end of the genomic-sized negative-strand template RNA (10) is used as a primer for subgenomic mRNA transcription at downstream intergenic regions (5). As a result, the leader RNA can be freely exchanged between mRNAs of different co-infecting viruses (18). Furthermore, the leader sequence evolves very rapidly during virus passages in tissue culture; specifically, the number of pentanucleotide (UCUAA) repeat sequences at the 3'-end of the leader decreases upon serial passages (15). The change of leader sequences also resulted in the change of subgenomic mRNA species synthesized (15). Thus, the leader sequence of MHV is very important for MHV mRNA transcription. However, it is not clear whether the free leader RNA species is also involved in MHV RNA replication.

The JHM strain of MHV (MHV-JHM) has been shown to generate defective-interfering (DI) RNAs during high-multiplicity passages $(14,19)$. MHV DI RNAs are classified into two types. One is DI RNA of nearly genomic size, which is efficiently packaged into virus particles and replicates itself even in the absence of helper virus infection (16). Another group are smaller DI RNAs of the classical type. One of these RNAs, DIssE, which is the smallest DI RNA detected, has been analyzed in grerater detail (16). DIssE RNA synthesis requires a helper virus and are inefficiently packaged into virus particles (16). Thus, it may lack packaging signals. On the other hand, since it is efficiently replicated in DI-infected cells, DIssE RNA must contain the sequences essential for viral RNA replication. Since DI RNAs replicate more efficiently than the standard MHV RNA (16), DI RNAs provide an opportunity for the study of MHV RNA replication. In this study, we show that MHV DI RNA replication involves a high-frequency, site-specific leader sequence switching, suggesting that a leader RNA supplied in trans is also utilized for MHV RNA replication. 


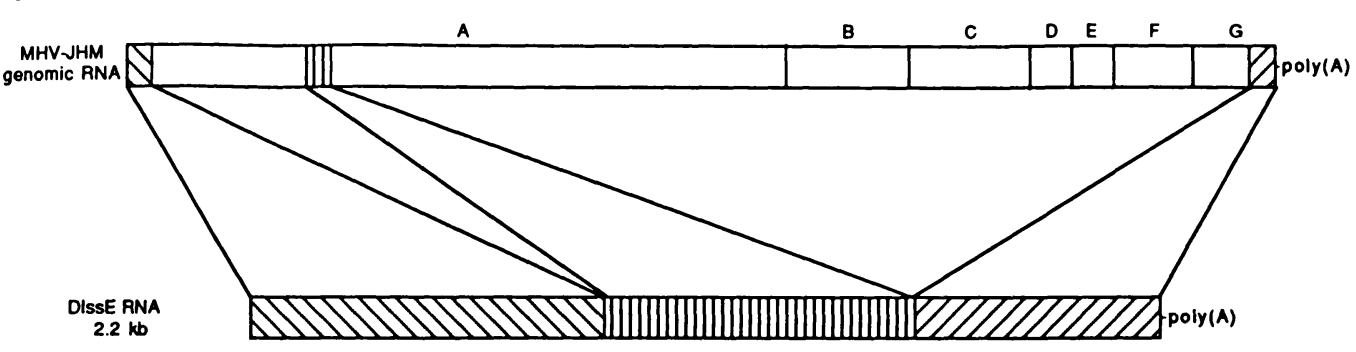

b
MHV-JHM
10
20
30
40
50
DIsSE (a) UAUAAGAGUGAAUGGCGUCCGUACGUACCCAAUCUACUCUAAAACUCUUG
DISSE (b) UAUAAGAGUGAAUGGCGUCCGUACGUACCCAAUCUACUCUAAAACUCUUG

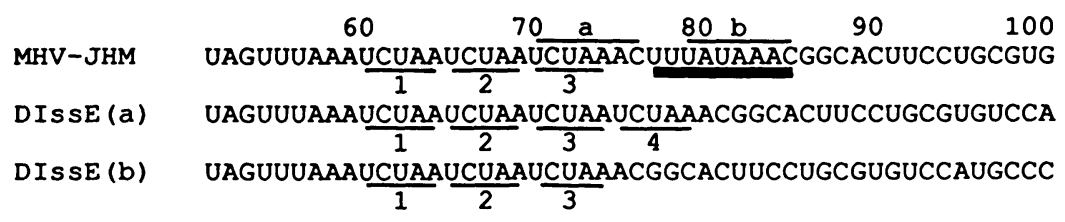

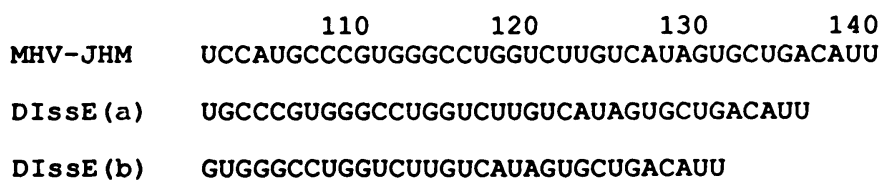

Figure 1. Diagram of the structure of DIssE RNA.

(a) A comparison between the sequence of DIssE RNA and that of standard MHV-JHM genomic RNA. A-G represent the seven genes of MHV (7).

(b) The 5'-end sequence of DIssE and MHV-JHM genomic RNA. The letters a and b represent the canonical seven-nucleotide sequence (22). A bold solid line represents the nine-nucleotide sequence which is deleted in DIssE but present in MHV-JHM. DIssE(a) and DIssE(b) represent the two species of DIssE RNA which have different number of UCUAA repeats. Sequence data were obtained from the primer extension analysis (data not shown). Three base substitutions are indicated by asteriks.

\section{MATERIALS AND METHODS}

Viruses and Cells. The plaque-cloned A59 strain of MHV (MHV-A59) was used as a helper virus. Mouse L2 cells and DBT cells were used for RNA transfection and propagation of viruses, respectively.

cDNA cloning of DIssE and DNA construction. cDNA cloning followed the general method of Gubler and Hoffman (4). Construction of various plasmids will be described elsewhere.

RNA transcription and transfection. Plasmid DNAs were linearized by $X b a$ I digestion and transcribed with T7 RNA polymerase as previously described (24). RNA transfection was done as previously described (16).

Preparation of virus-specific intracellular RNA and agarose gel electrophoresis. Virus-specific RNAs in RNAtransfected cells or virus-infected cells were labeled with $32 \mathrm{P}$-orthophosphate as previously described (20). Virus-specific RNA was separated by electrophoresis on $1 \%$ agarose gels after denaturation with 1M glyoxal (21). Preparative gel electrophoresis in $1 \%$ agarose gels containing $6 \mathrm{M}$ urea was performed as previosly described (19).

RNA sequencing. Dideoxynucleotide chain termination methods adapted for RNA sequencing was used (26). 


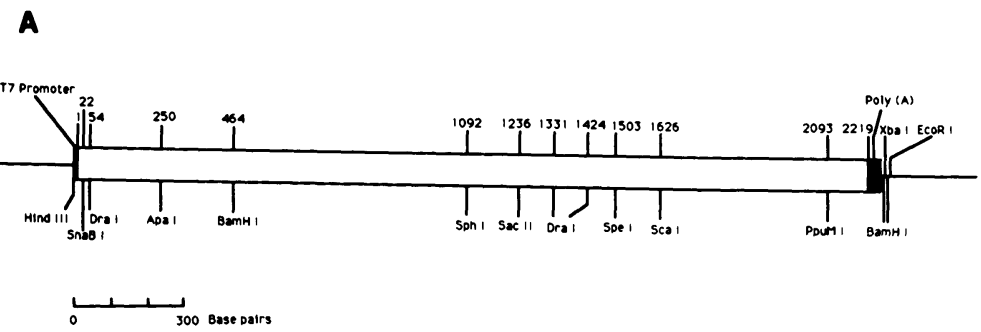

B

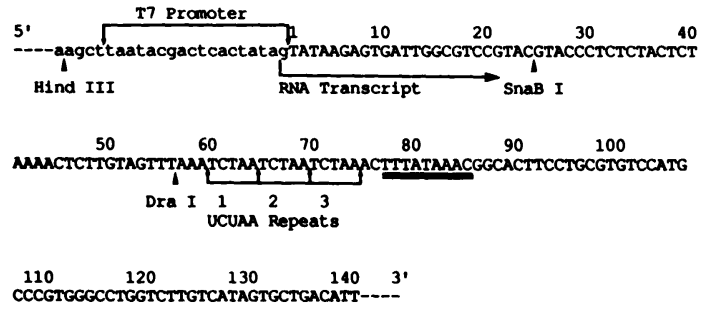

C

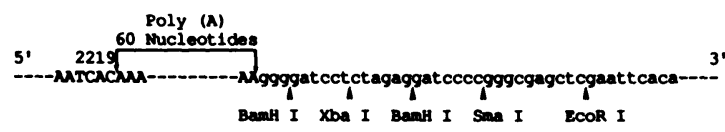

Figure 2. Diagram of the structure of DE5-w3.

(A) Open box indicates DI cDNA sequence, and solid lines indicate vector sequences.

(B) Sequence of the 5'-end region of DI CDNA of DE5-w3. The nine-nucleotide sequence is underlined. The DI sequences are in capital letters while vector sequences are in lower cases.

(C) Sequence of the 3'-end region of DI CDNA of DE5-w3 and plasmid polylinker sites.

\section{RESULTS}

cDNA cloning and sequencing of DIssE RNA. To understand the primary structure of DIssE RNA, cDNA clones were generated from gel-purified DIssE RNA using oligo(dT) as a primer. A diagram representing the structure of DIssE RNA and that of MHV-JHM genomic RNA are shown in Figure 1. Sequence analysis of DIssE cDNA clones revealed that DIssE RNA consists of three different regions of MHV-JHM genomic RNA. The first region represents 864 nucleotides from the 5'-end of the genomic RNA (24). The second region, 748 nucleotides in length, is a region within gene 1 that corresponds to the region at 3.3 to $4 \mathrm{~kb}$ from the 5'-end of genomic RNA (Baker et al., in press), and the third region contains a sequence of 601 nucleotides derived from the extreme 3'-end of the genomic RNA (23). The entire sequence of DIssE RNA is identical to that of the corresponding regions of MHV genomic RNA, with some exceptions in the leader sequence region, where 3 bases were substituted and nine nucleotides (UUUAUAAAC) were deleted in DIssE at the junction between the leader RNA and the remaining genomic sequences. Also the 5'-end of DIssE RNA was heterogeneous with respect to the number of UCUAA repeats within the leader sequence (Figure 1B).

Construction of DI RNAs capable of efficient replication in the presence of helper virus. To utilize DI RNAs for understanding the mechanism of MHV RNA synthesis and to assess the role of the 9 nucleotides at the junction between leader and the remaining genome sequence, we have constructed a complete DI CDNA clone $(2.2 \mathrm{~kb})$ which contains the complete leader sequence of MHV-JHM plus the 9 nucleotides. This DI sequence was placed downstream to the promoter for T7 RNA polymerase, so that one additional $\mathrm{G}$ residue is added to the 5 '-end of the run-off RNA transcript (DE5-w3) (Figure 2). The 3'-end of this DI cDNA construct contains a poly (A) sequence, which is followed by a polylinker sequence (Figure 2). The plasmid was linearized by $X b a$ $\mathrm{I}$, and transcribed by T7 RNA polymerase in the presence of a cap analogue $\left(\mathrm{m}^{7} \mathrm{G}\left(5^{\prime}\right) \mathrm{ppp}\left(5^{\prime}\right) \mathrm{G}\right)$. The RNA was transfected into monolayers of mouse L2 cells which had been infected with the A59 strain of MHV (MHVA59), $1 \mathrm{hr}$ prior to transfection. MHV-A59 can be distinguished from MHV-JHM in both leader and genomic sequences $(6,22)$. Virus-specific RNAs were labeled with ${ }^{32} \mathrm{P}$-orthophosphate in the presence of actinomycin D (20), and analyzed by agarose gel electrophoresis. As shown in Figure 3, DI RNA of $2.2 \mathrm{~kb}$ was detected as early as $6 \mathrm{hrs}$ after transfection. When virus harvested from transfected cells (passage 1 virus) was used to infect 


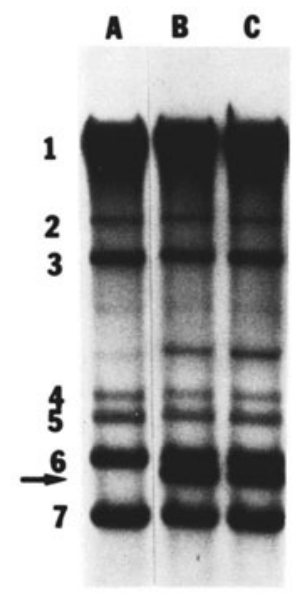

Figure 3. Intracellular RNA synthesis after transfection with DE5-w3 RNA and infection with MHV-A59. Lane A: mock-transfected and MHV-A59-infected cells, Lane B: DE5-w3-transfected and MHV-A59-infected cells, Lane C: passage 1 virus-infected cells. The arrow indicates the DI RNA.

L2 cells, the DI RNA was also detected in infected cells. This result indicates that this RNA could be packaged into virus particles. These data thus demonstrated that in vitro synthesized DE5-w3 RNA replicated efficiently in transfected cells in the presence of a heterologous helper virus, MHV-A59.

Change of 5'-end sequence of DI RNA in transfected cells. Since the leader sequences of mRNAs can be exchanged freely between different viruses during co-infection (18) and the leader sequence evolves rapidly during virus passages in tissue culture (15), we examined whether any alteration occurred at the 5'-end of DI RNA species in transfected cells. For this study, we have prepared several DI cDNA constructs which have different sequences within the leader sequence. The 5'-end structures of the transcripts are shown in Figure 4. The DI RNAs transcribed from these constructs were transfected into L2 cells as described above. The 5'-end sequence of gel-purified DI RNAs obtained from transfected cells were examined by RNA sequencing, using a primer complementary to nucleotides $172-188$ from the 5' end of the genomic RNA. Results of RNA sequencing are shown in Figure 4. Surprisingly, all the DI RNA isolated from RNA-transfected-cells contained an A at position 35 and two repeats of the UCUAA sequence, which is identical to the leader sequence of MHV-A59 (6). All DI RNAs had a U at nucleotide 111, identical to parent MHV-JHM (22), whereas MHV-A59 had a C at this position, indicating that the leader sequence of DI RNA was replaced by the leader sequence of the helper virus MHV-A59.

To examine whether the sequence alteration was limited to the 5 -end of DI RNA or involved the entire DI genome, we examined the structure of DI RNA by T1-oligonucleotide fingerprinting. The fingerprinting analysis of the in vitro synthesized DE5-w3 RNA and the DI RNA isolated from the DE5-w3 RNA-transfected cells demonstrated that sequence alteration occurred only at the leader sequence and the rest of sequence did not undergo gross sequence alteration (data not shown). We therefore concluded that all of the DI RNAs switched the leader sequence with the helper virus.

A nine-nucleotide deletion eliminated the leader RNA switching.

We have previously failed to detect leader RNA switching between a naturally occurring DI RNA and MHV-A59 (16). Since the naturally occurring DI RNA has a deletion of nine nucleotides at the junction between the leader and the remaining genomic sequence and has three nucleotide substitutions within leader sequence as compared to the standard MHV-JHM (22), we speculated that these sequence differences between the naturally occurring DI RNA and the in vitro constructed RNAs used here might have accounted for the difference in the leader RNA switching. Therefore we prepared three different DI cDNA constructs which contained a deletion of the nine nucleotides. As shown in Figure 5, the DI RNAs isolated from cells transfected with these DI RNA constructs retained the leader sequence of the input DI RNAs. Since the only difference between DE-1A and DE5-w4, and between DE-2c and DE107-w4 RNAs is the absence or presence of the nine nucleotides, we concluded that the presence of the nine nucleotides located at the junction between the leader and the remaining DI sequence is crucial for the leader sequence exchange with the helper virus during DI RNA replication. 


\begin{tabular}{|c|c|c|c|c|}
\hline $\begin{array}{l}\text { In viero } \\
\text { DI RNA } \\
\text { transcripes }\end{array}$ & & & $\begin{array}{l}\text { IUmber o } \\
\text { UncunA } \\
\text { repeats }\end{array}$ & \\
\hline DES-w4 & $\mathbf{U}$ & $\mathbf{U}$ & $4 R$ & $\overline{\mathbf{U}}$ \\
\hline DE5-w3 & $\mathrm{U}$ & $\mathrm{U}$ & $3 R$ & $\bar{U}$ \\
\hline DE5-w1 & $\mathrm{U}$ & $\mathbf{U}$ & IR & $\bar{U}$ \\
\hline DE107-w4 & $\mathbf{A}$ & $\mathbf{U}$ & $4 \mathrm{R}$ & $\overline{\mathrm{U}}$ \\
\hline DE107-w3 & A & $\mathbf{U}$ & $3 R$ & $\mathbf{U}$ \\
\hline DE107-w1 & A & $\mathbf{U}$ & $1 \mathbf{R}$ & $\mathrm{U}$ \\
\hline
\end{tabular}

MHV-A59 \begin{tabular}{|lllll}
\hline U & A & 2R & C \\
\hline
\end{tabular}

\begin{tabular}{|c|c|c|c|c|}
\hline $\begin{array}{l}\text { In vivo } \\
\text { DI RNAs }\end{array}$ & $\mathbf{U}$ & A & $2 \mathbf{R}$ & $\mathbf{U}$ \\
\hline $\begin{array}{l}\text { Nuclooside } \\
\text { posidion }\end{array}$ & 12 & 35 & & 111 \\
\hline
\end{tabular}

Figure 4. The schematic representation of the 5'-end sequences of DI RNAs synthesized in vitro and obtained from DI RNA-transfected cells.

Only the diverged nucleotides are indicated. $1 R, 2 R, 3 R$ and $4 R$ represent one, two, three and four repeats of UCUAA sequence, respectively. The nine-nucleotide sequences (UUUAUAAAC) are represented as crosshatched boxes.

\section{DISCUSSION}

The sequence analysis of DIssE RNA revealed that it is composed of three discontiguous parts of the viral genome, including the 5'-end and 3'-end of genomic RNA. Our previous study has demonstrated that DIssE is replicated from its negative template in the presence of a helper virus (16). Therefore, the DIssE sequence likely contains essential recognition signals for MHV replication. The structure of DIssE RNA supports the likelihood that the recognition signals for the synthesis of negative-strand RNA and positive-strand RNA are localized at the 3'-end and 5'-end of genomic RNA, respectively.

We have also demonstrated that the leader sequence of DI RNAs switched to that of the helper virus during DI RNA replication. This leader sequence switching requires the presence of a nine-nucleotide sequence at the junction between leader RNA and the remaining genomic sequence. Since only one round of virus replication was required for the leader RNA switching and the majority of RNA in the DI RNA-transfected cells acquired the leader RNA of the helper virus, this leader RNA switching must have occurred very early in the DI replication process and at a very high frequency. This result is most consistent with the interpretation that a free leader RNA derived from the helper virus is involved in the replication of DI RNA. We have previously shown that leader RNA species can be freely exchanged between subgenomic mRNAs of two MHVs during mixed infection (18). These two studies suggest that both transcription and replication of MHV RNAs involve a free leader RNA species.

The MHV DI RNAs constructed here showed an extremely high efficiency of RNA replication. Considering the low efficiency of RNA transfection, this finding indicates that the DI RNA has a much higher efficiency of RNA replication than the mRNAs from helper viruses. This replication rate is even higher than that of comparable DI RNA of Sindbis virus, which requires several cycles of virus passages before DI RNAs could be detected (13). Thus, MHV DI RNA could be a potentially useful vector for expressing foreign genes in mammalian cells. It should be noted that in either DI RNA-transfected or passage 1 virus-infected cells, the helper virus RNA synthesis was not inhibited. This was probably due to the low efficiency of RNA transfection and lack of specific packaging signals in these DI RNAs. We have previously shown that DIssE RNA is packaged into virion particles nonspecifically and at a very low efficiency (16). Nevertheless, whatever small amount of RNA packaged apparently is enough to replicate into a major RNA species. The addition of a packaging signal to these DI cDNA constructs could further increase its utility as an expression vector. 


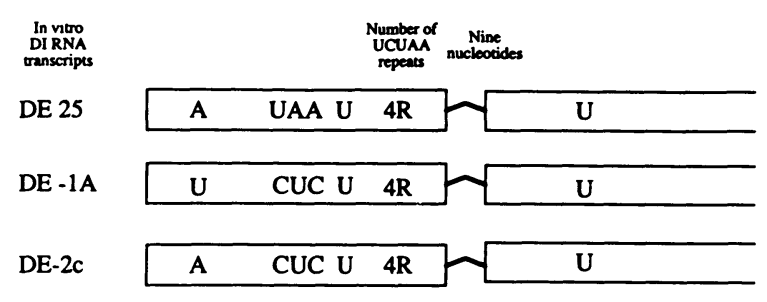

\begin{tabular}{lllll} 
MHV-A59 & U & CUC A & $2 R$ & C \\
\hline
\end{tabular}

\begin{tabular}{|c|c|c|c|c|}
\hline DE 25 & $\mathbf{A}$ & UAA U & $4 R$ & $\mathrm{U}$ \\
\hline DE-1A & $\mathrm{U}$ & CUC U & $4 R$ & $\mathrm{U}$ \\
\hline DE-2c & A & CUC U & $4 R$ & $\mathrm{U}$ \\
\hline $\begin{array}{c}\text { Nucleotide } \\
\text { postion }\end{array}$ & 12 & 30313235 & & 111 \\
\hline
\end{tabular}

Figure 5. The schematic representation of the 5'-end sequences of DI RNAs containing a nine-nucleotide deletion.

$2 R$ and $4 R$ represent the two and four repeats of UCUAA sequence, respectively. The nine-nucleotide sequence in MHV-A59 is represented by a cross-hatched box. The deletion of the nine-nucleotide is shown as a thin line. The nucleotide sequences which are not denoted in the diagram share the same sequence as that of DE5-w3 (see Figure 2B).

\section{REFERENCES}

1. Baric, R. S., Shieh, C.-K., Stohlman S.A., and Lai, M. M. C. (1987). Analysis of intracellular small RNAs of mouse hepatitis virus: Evidence for discontinuous transcription. Virology 156, 342-354.

2. Baric, R. S., Stohlman, S. A., and Lai, M. M. C. (1983). Characterization of replicative intermediate RNA of mouse hepatitis virus: Presence of leader RNA sequences on nascent chains. J. Virol. 48, 633-640.

3. Baric, R. S., Stohlman, S. A., Razavi, M. K., and Lai, M. M. C. (1985). Characterization of leader-related small RNAs in coronavirus-infected cells: Further evidence for leader-primed mechanism of transcription. Virus Res. 3, 19-33.

4. Gubler, U., and Hoffman, B. J. (1983). A simple and very efficient method for generating cDNA libraries. Gene 25, 263-269.

5. Lai, M. M. C. (1986). Coronavirus leader-RNA-primed transcription: an alternative mechanism to RNA splicing. BioEssays 5, 257-260.

6. Lai, M. M. C., Baric, R. S., Brayton, P. R., and Stohlman, S. A. (1984). Characterization of leader RNA sequences on the virion and mRNAs of mouse hepatitis virus, a cytoplasmic RNA virus. Proc. Natl. Acad. Sci. USA $81,3626-3630$.

7. Lai, M. M. C., Brayton, P. R., Armen, R. C., Patton, C. D., Pugh, C., and Stohlman, S. A. (1981). Mouse hepatitis virus A59: mRNA structure and genetic localization of the sequence divergence from hepatotropic strain MHV-3. J. Virol. 39, 823-834.

8. Lai, M. M. C., Makino, S., Soe, L. H., Shieh, C.-K., Keck, J. G., and Fleming, J. O. (1987). Coronavirus: A jumping RNA transcription. Cold Spring Harbor Symposia on Quantitative Biology, Vol. LII, 359-365.

9. Lai, M. M. C., Patton, C. D., Baric, R. S., and Stohlman, S. A. (1983). Presence of leader sequences in the mRNA of mouse hepatitis virus. J. Virol. 46, 1027-1033. 
10. Lai, M. M. C., Patton, C. D., and Stohlman, S. A. (1982). Replication of mouse hepatitis virus: Negative-stranded RNA and replicative form RNA are of genome length. J. Virol. 44, 487-492.

11. Lai, M. M. C., and Stohlman, S. A. (1978). RNA of mouse hepatitis virus. J. Virol. 26, 236-242.

12. Leibowitz, J. L., Wilhelmsen, K. C., and Bond, C. W. (1981). The virus-specific intracellular RNA species of two murine coronavirus: MHV-A59 and MHV-JHM. Virology 114, 39-51.

13. Levis, R., Weiss, B. G., Tsiang, M., Huang, H. and Schlesinger, S. (1986). Deletion mapping of Sindbis virus DI RNAs derived from cDNAs defines the sequences essential for replication and packaging. Cell 44, 137145.

14. Makino, S., Fujioka, N., and Fujiwara, K. (1985). Structure of the intracellular defective viral RNAs of defective interfering particles of mouse hepatitis virus. J. Virol. 54, 329-336.

15. Makino, S., and Lai, M. M. C. (1989). Evolution of the 5'-end of genomic RNA of murine coronaviruses during passages in vitro. Virology 169, 227-232.

16. Makino, S., Shieh, C.-K., Keck, J. G., and Lai, M. M. C. (1988). Defective-interfering particles of murine coronaviruses: Mechanism of synthesis of defcctive viral RNAs. Virology 163, 104-111.

17. Makino, S., Soe, L. H., Shieh, C.-K., and Lai, M. M. C. (1988). Discontinuous transcription generates heterogeneity at the leader fusion sites of coronavirus mRNAs. J. Virol. 62, 3870-3873.

18. Makino, S., Stohlman, S. A., and Lai, M. M. C. (1986). Leader sequences of murine coronavirus mRNAs can be freely reassorted: Evidence for the role of free leader RNA in transcription. Proc. Natl. Acad. Sci. USA $83,4204-4208$.

19. Makino, S., Taguchi, F., and Fujiwara, K. (1984). Defective interfering particles of mouse hepatitis virus. Virology 133, 9-17.

20. Makino, S., Taguchi, F., Hirano, N., and Fujiwara, K. (1984). Analysis of genomic and intracellular viral RNAs of small plaque mutants of mouse hepatitis virus, JHM strain. Virology 139, 138-151.

21. McMaster, G. K., and Carmichael, G. G. (1977). Analysis of single- and double-stranded nucleic acids on polyacrylamide and agarose gels by using glyoxal and acridine orange. Proc. Natl. Acad. Sci. USA 74, 48354838 .

22. Shieh, C.-K., Soe, L. H., Makino, S., Chang, M.-F., Shohlman, S. A., and Lai, M. M. C. (1987). The 5 -end sequence of the murine coronavirus genome: Implications for multiple fusion sites in leader-primed transcription. Virology 156, 321-330.

23. Skinner, M. A., and Siddell, S. G. (1983). Coronavirus JHM: Nucleotide sequence of the mRNA that encodes nucleocapsid protein. Nucleic Acid Res. 15, 5045-5054.

24. Soe, L. H., Shieh, C.-K., Baker, S. C., Chang, M.-F., and Lai, M. M. C. (1987). Sequence and translation of the murine coronavirus 5'-end genomic RNA reveals the N-terminal structure of the putative RNA polymerase. J. Virol. 61, 3968-3976.

25. Spaan, W., Delius, H., Skinner, M., Armstrong, J., Rottier, P., Smeekens, S., van der Zeijst, B. A. M., and Siddell, S. G. (1983). Coronavirus mRNA synthesis involves fusion of non-contiguous sequences. EMBO J. 2, 1939-1944.

26. Zimmern, D., and Kaesburg, P. (1978). 3'-terminal nucleotide sequence of encephalomyocarditis virus RNA determined by reverse transcriptase and chain terminating inhibitors. Proc. Natl. Acad. Sci. USA 75, 4257-4261. 